\title{
Dynamic fragmentation of a spinor Bose-Einstein condensate
}

\author{
Ö. E. Müstecaplı̆ğlu \\ Koç University, Rumelifeneri Yolu, 34450 Sarlyer, Istanbul, Turkey \\ M. Zhang, S. Yi, and L. You \\ School of Physics, Georgia Institute of Technology, Atlanta, Georgia 30332-0430, USA \\ C. P. Sun \\ Institute of Theoretical Physics, Chinese Academy of Science, Beijing 100080, People's Republic of China
}

(Received 31 July 2003; published 23 December 2003)

\begin{abstract}
We investigate dynamical behavior of fragmentation of a spinor Bose-Einstein condensate in both the semiclassical and quantum regimes. We show that an unfragmented condensate can become fragmented at later times due to the inherent interactions of elastic atomic collisions, when a certain phase relationship exists among the state amplitudes in different components initially. A close relationship between the fragmentation and population dynamics of atomic distributions within different spin components is found for certain initial states with zero magnetization. The type of fragmentation regime is characterized via atom-number fluctuations. We also address complications of our study when the condensate spatial mode function is different for each spin component.
\end{abstract}

DOI: $10.1103 /$ PhysRevA.68.063616

PACS number(s): $03.75 . \mathrm{Mn}, 03.75 . \mathrm{Kk}, 03.75 . \mathrm{Nt}$

\section{INTRODUCTION}

The ground-state properties of an interacting atomic Bose-Einstein condensate (BEC) are of fundamental importance to the understanding of trapped quantum gases [1]. According to the orthodox picture of Bose-Einstein condensation, the appearance of a condensate is accompanied by the emergence of one macroscopic eigenvalue of the singleparticle density matrix [1], and the corresponding eigenvector is the single-particle orbital to which all atoms condense. When more than one eigenvalue of the single-particle density matrix becomes macroscopic, it becomes impossible to assign a single-particle wave function (or orbital) to the condensate. In this case the condensate is said to be fragmented [2], and it can be effectively viewed as a Schrödinger cat state of a macroscopic superposition of condensed atoms in more than one orbital.

A condensate of spin-zero particles with repulsive and local interactions is known to be unfragmented according to the arguments by Nozières and James [2]. Attractive atomatom interaction alone does not lead to fragmentation either, as a fragmented state is generally believed to occur when the number of atoms is higher than the stability limit of the condensate. In other words, an attractive condensate always collapses before fragmentation occurs. It is difficult for a bosonic system to achieve a fragmented ground state because of the well-known argument [2]: while the Hartree energy is the same whether a condensate is fragmented or not, the exchange energy arising from the symmetrization usually increases for a fragmented state, thus making it less favorable to become ground state. On the other hand, by considering nonlocal interactions in an inhomogeneous environment such as those afforded by a Josephson-junction type potential, the exchange interaction can be kept small, thus leading to the possibility of a spatially fragmented state, which of course needs to be distinguished from a fragmentation with respect to the internal degrees of freedom.

Recently, it was argued that a fragmented ground state does occur in an optically trapped spin-1 condensate [3]. Such a vectorial condensate can be produced either by transferring a condensate (of atoms with nonzero hyperfine spins) from a magnetic trap into an optical trap [4], or by evaporative cooling of atoms through the all optical route [5]. For both ${ }^{23} \mathrm{Na}$ [4] and ${ }^{87} \mathrm{Rb}$ [5] atoms, condensation usually occurs in the lowest hyperfine state with $f=1$, i.e., with three internal Zeeman states of $m_{f}=+1,0,-1$. Since the lowenergy $s$-wave atomic collisions lead to an interaction that is rotationally invariant, the order parameter for such a spinor condensate contains three components [6-10], where earlier studies have uncovered a host of complex ground-state structures that can exhibit novel dynamics [3,11,12]; e.g., fragmentation [2], spin mixing, and entanglement $[9,6,12,13]$. In particular, we note that a transition to a fragmented condensate for repulsive pairing interaction can be achieved by control of optical trap parameters, thus effectively realizing a quantum phase transition [14].

In this study, we explore optically controlled fragmentation of a spin-1 condensate. Due to the presence of internal degrees of freedom, fragmentation can occur even in a homogeneous environment. The suppression of exchange interaction arises from the exchanges among atoms in different Zeeman states, and the corresponding eigenfunctions of the single-particle density matrix with macroscopic eigenvalues are highly correlated states, thus potentially useful for studies of quantum correlation and entanglement [12]. Our objective is to engineer a fragmented condensate using external laser pulses Raman coupled to single atom transitions, as in spin mixing phenomena studied earlier [12]. We propose to control the fragmentation through the amplitudes and phase differences of Raman pulses, and we show that this is indeed possible and leads to detectable effects in individual Zeeman level atom population oscillations for such initial prepara- 
tions. To characterize the type of fragmentation regime, we study two-particle correlation functions and we find that our fragmented condensate system behaves similar to a condensate in the coherent-fragmented regime studied earlier [3].

This paper is organized as follows. We first describe our system of an optically trapped spin-1 BEC in Sec. II. A simple three-mode model is then developed in Sec. III. Following these formulations, we explore the fragmentation dynamics first semiclassically and then quantum mechanically in Secs. IV and V, respectively. Finally we conclude in Sec. VI.

\section{OUR PHYSICS SYSTEM}

Our system consists of $N$ spin-1 atoms interacting via $s$-wave elastic collisions as appropriate for either ${ }^{23} \mathrm{Na}$ or ${ }^{87} \mathrm{Rb}$. It is assumed that the atoms are confined in the $f=1$ hyperfine manifold within a far-off-resonant optical dipole trap [4,5]. A spin-1 condensate is therefore described by a three-component order parameter $\psi(\vec{x})$ $=\left[\psi_{+}(\vec{x}), \psi_{0}(\vec{x}), \psi_{-}(\vec{x})\right]$. This vectorial nature allows for a rotationally invariant Hamiltonian description as required for $s$-wave collisions, and the two-body interaction is given by $V\left(\vec{r}_{1}-\vec{r}_{2}\right)=\delta\left(\vec{r}_{1}-\vec{r}_{2}\right)\left(g_{0} \mathcal{P}_{0}+g_{2} \mathcal{P}_{2}\right)=\delta(\vec{r})\left(\lambda_{0}+\lambda_{2} \vec{F}_{1} \cdot \vec{F}_{2}\right)$ $[6-8,10]$, where $\mathcal{P}_{0}$ and $\mathcal{P}_{2}$ denote, respectively, the projections into the symmetrical channels of $F=0$ and 2 of the total spin $\vec{F}=\vec{f}_{1}+\vec{f}_{2}$. In terms of their respective scattering lengths $a_{0}$ and $a_{2}$ and with $g_{f}=4 \pi \hbar^{2} a_{f} / M$ (atomic mass $M)$, one finds that $\lambda_{0}=\left(g_{0}+2 g_{2}\right) / 3$ and $\lambda_{2}=\left(g_{2}-g_{0}\right) / 3$ $[6,9]$. According to atomic theory, $\left|\lambda_{0}\right| \gg\left|\lambda_{2}\right|$ for both ${ }^{23} \mathrm{Na}$ or ${ }^{87} \mathrm{Rb}$ atoms. Therefore, it is usually convenient to express the total system Hamiltonian in two separate parts [9],

$$
H=\int d \vec{r}\left[\mathcal{H}_{s}(\vec{r})+\mathcal{H}_{a}(\vec{r})\right]
$$

a symmetric part $H_{s}$ and an asymmetric part $H_{a}$ with the corresponding Hamiltonian density

$$
\begin{aligned}
\mathcal{H}_{s}(\vec{r})= & \psi_{i}^{\dagger}\left(-\frac{\hbar^{2} \nabla^{2}}{2 M} \delta_{i j}+V_{\mathrm{t}}^{i j}\right) \psi_{j}+\frac{\lambda_{0}}{2} \psi_{i}^{\dagger} \psi_{j}^{\dagger} \psi_{i} \psi_{j}, \\
\mathcal{H}_{a}(\vec{r})= & \frac{\lambda_{2}}{2}\left[\psi_{+}^{\dagger} \psi_{+}^{\dagger} \psi_{+} \psi_{+}+\psi_{-}^{\dagger} \psi_{-}^{\dagger} \psi_{-} \psi_{-}\right. \\
& +2 \psi_{0}^{\dagger} \psi_{0}\left(\psi_{+}^{\dagger} \psi_{+}+\psi_{-}^{\dagger} \psi_{-}\right)-2 \psi_{+}^{\dagger} \psi_{+} \psi_{-}^{\dagger} \psi_{-} \\
& \left.+\left(\psi_{+}^{\dagger} \psi_{-}^{\dagger} \psi_{0}^{2}+\text { H.c. }\right)\right],
\end{aligned}
$$

where a summation over repeated indices $i, j= \pm, 0$ is implied. $V_{\mathrm{t}}(\vec{r})$ is the external confinement, assumed to be internal state independent as for a far off-resonant optical trap. In the above, terms involving a single spin component describe self-scattering, while two-component terms describe various cross-scattering processes, and terms with all three components are responsible for spin mixing.

A straightforward approach to determine the system dynamics would be to solve for the Heisenberg operator equation of motion $i \hbar \partial \psi_{i} / \partial t=\left[\psi_{i}, H\right]$. This is usually impos- sible because the nonlinear interaction terms lead to a coupled chain of an infinite hierarchy of moments for the system operators. To overcome this difficulty, one can adopt the mean-field approximation and simply replace the Schrödinger field operators by $c$ numbers at the cost of neglecting quantum fluctuations. This results in three coupled GrossPitaevskii equations GPEs) that can be studied with numerical techniques [15]. To study the effect of quantum fluctuations, one then implements the widely used Bogoliubov approximation by taking $\psi=\langle\psi\rangle+\delta \psi$, i.e., supplementing the $c$-number part with the fluctuation operator $\delta \psi$ and keeping its quadratic terms in the Hamiltonian [16].

Previous studies have revealed that even classical fluctuations in the $c$-number condensate fields can lead to modulation instabilities for a ferromagnetically interacting condensate [17]. In this paper, we will focus on atoms with antiferromagnetic interactions where modulation instabilities are not predicted to complicate the system dynamics. Instead of adopting the previously described procedure with the use of the Bogoliubov approximation to explore quantum dynamics, we will take an alternative approach by focusing on an appropriate choice of basis states $\left\{\phi_{n}(\vec{r})\right\}$ for the expansion $\psi_{i}=\Sigma_{n} a_{n} \phi_{n}$. Several choices have been made before, including the use of a plane-wave basis which assumes a translational symmetry [18]; a harmonic-oscillator basis which assumes weak atom interactions $\lambda_{2,0}[19-21]$; and the use of mode functions of the three coupled mean-field GPEs which assumes a small $\lambda_{2}[15,22]$. The last choice is widely termed the single-mode approximation (SMA) [9] as the mode functions are assumed identical for all three spin components. This approximation has been remarkably successful because current spin- 1 condensed atoms strictly satisfy the condition of $\left|\lambda_{0}\right| \gg\left|\lambda_{2}\right|$, thus one can even approximate the identical mode function with the solution of a scalar GPE of an interaction coefficient $\lambda_{0}$. However, this approximation does break down for larger number of atoms $\left(N \sim 10^{4}\right)$ with typical trap parameters $[15,23]$. The harmonic oscillator basis, on the other hand, corresponds to noninteracting atoms, so can only be used with smaller number of atoms $N<10^{3}$ [21].

In this study, we will not use the SMA. Instead, we shall use the self-consistent mode functions from the coupled GPE to perform quantum dynamical studies. Although we still employ frozen spatial mode functions, we can determine them numerically without neglecting any term in the Hamiltonian. As a result, we can accommodate different mode functions for different condensate components, i.e., we do not have to use the SMA. Substituting $\psi_{i}=a_{i} \phi_{i}$ into the Hamiltonian (1), we get a three-mode model described by

$$
\begin{gathered}
H_{s}=E_{i} a_{i}^{\dagger} a_{i}+\frac{g_{i j}^{s}}{2} a_{i}^{\dagger} a_{j}^{\dagger} a_{i} a_{j}, \\
H_{a}=\frac{g_{i i}^{a}}{2} n_{i}\left(n_{i}-1\right)+g_{+0}^{a} n_{+} n_{0}+g_{-0}^{a} n_{-} n_{0}-g_{+-}^{a} n_{+} n_{-} \\
+\left(g a_{+}^{\dagger} a_{-}^{\dagger} a_{0}^{2}+\text { H.c. }\right),
\end{gathered}
$$

where $n_{i}=a_{i}^{\dagger} a_{i}$, and the overlap integral terms are given by 


$$
\begin{gathered}
E_{i}=\int d \vec{r} \phi_{i}^{*}\left(-\frac{\hbar^{2} \nabla^{2}}{2 M} \delta_{i j}+V_{\mathrm{t}}^{i j}\right) \phi_{j}, \\
g_{i j}^{s}=\lambda_{0} \int d \vec{r}\left|\phi_{i}\right|^{2}\left|\phi_{j}\right|^{2}, \\
g_{i j}^{a}=\frac{\lambda_{2}}{\lambda_{0}} g_{i j}^{s}, \\
g=\lambda_{2} \int d \vec{r} \phi_{+}^{*} \phi_{-}^{*} \phi_{0}^{2} .
\end{gathered}
$$

For a spin-independent trap, $V_{t}^{i j}=V_{t} \delta_{i j}$, thus the only interaction coefficient that can take on a complex value is $g$ as given above. Within the mean-field approximation, we denote the ground-state condensate order parameter as $\phi_{j}$ $=\left|\phi_{j}\right| \exp \left(i \delta_{j}\right)$. The associated phase factor for $g$ is then given by $\delta=\delta_{+}+\delta_{-} 2 \delta_{0} \cdot g$ becomes real when $\bmod (\delta)$ $=0$ or $\pi$, one of the conditions required to have stationary states in the coupled GPE [24].

Within the SMA [12], the condensate wave function $\phi(\vec{r})$ is determined by the GPE,

$$
\left[-\frac{\hbar^{2}}{2 M} \nabla^{2}+V_{\mathrm{t}}(\vec{r})+\lambda_{0} N|\phi|^{2}\right] \phi(\vec{r})=\mu \phi(\vec{r})
$$

(see, however, Ref. [23]), where $\mu$ is the chemical potential. Following earlier studies $[6,9]$, we define angular momentum like operator $L$ according to

$$
\begin{gathered}
L_{+}=\sqrt{2}\left(a_{+}^{\dagger} a_{0}+a_{0}^{\dagger} a_{-}\right), \\
L_{-}=L_{+}^{\dagger}, \\
L_{z}=n_{+}-n_{-},
\end{gathered}
$$

and denote the total atom number by $N=n_{+}+n_{-}+n_{0}$, then the system Hamiltonian (3) takes on the simple form [9]

$$
H=\mu N-\lambda_{s} N(N-1)-2 \lambda_{a} N+\lambda_{a} L^{2}
$$

with $\lambda_{a, s}=\left(\lambda_{2,0} / 2\right) \int d \vec{r}|\phi(\vec{r})|^{4}$. In this case, the su(2) algebra of the angular momentum operator allows for a simplified view of the eigenvalues and eigenstates. When the SMA breaks down, the eigensystem as described by the Hamiltonian (3) is complicated. However, as we show below, by enhancing the symmetry group to $\mathrm{SU}(3)$, several technical and conceptual advances can be achieved, and we find that condensate component populations, corresponding to diagonal elements of the single-particle density matrix, continue to exhibit dynamical spin mixing effects [9,12]. The offdiagonal elements, on the other hand, influence quantum correlations such as spin squeezing [25], entanglement [13], and fragmentation [6]. To understand the system quantum dynamics, it is therefore essential to determine the evolution of the complete single-particle density matrix within a quantum-mechanical framework.

\section{THREE-MODE MODEL}

In this section, we present our formulation for investigating the quantum dynamics of the coupled three-mode model. We note that the single-particle density matrix can be expressed in terms of the SU(3) generators of the general threemode Hamiltonian (3). This points to a systematic approach to examine the quantum dynamics of the density-matrix elements. We introduce auxiliary operators $U, V, T$ such that $a_{ \pm, 0}$ can be used similar to a Schwinger boson representation for $\mathrm{su}(3)$ Lie algebra in the following manner:

$$
\begin{gathered}
T_{+}=a_{+}^{\dagger} a_{-}, \quad T_{3}=\frac{1}{2}\left(n_{+}-n_{-}\right), \\
V_{+}=a_{+}^{\dagger} a_{0}, \quad V_{3}=\frac{1}{2}\left(n_{+}-n_{0}\right), \\
U_{+}=a_{-}^{\dagger} a_{0}, \quad U_{3}=\frac{1}{2}\left(n_{-}-n_{0}\right), \\
N=n_{+}+n_{-}+n_{0}, \quad Y=\frac{1}{3}\left(n_{+}+n_{-}-2 n_{0}\right) .
\end{gathered}
$$

Both $N$ are $T_{3}$ are constants of motion, corresponding, respectively, to the total number of atoms and one half of the system magnetization. The linear combinations in the form $X_{ \pm} \pm X_{\mp}$ for $X=T, U, V$, together with $T_{3}$ and $Y$ constitute a set of eight generators for $\mathrm{SU}(3)$, the spherical representation [26]. It can be seen immediately that $T_{ \pm, 3}$ as well as $U_{ \pm, 3}$ and $V_{ \pm, 3}$ fulfill $\mathrm{SU}(2)$ commutation relations $\left[X_{+}, X_{-}\right]$ $=2 X_{3}$ and $\left[X_{3}, X_{ \pm}\right]=X_{ \pm}$, the basis of angular momentum algebra. In terms of these operators we express angular momentum as $L_{+}=\sqrt{2}\left(V_{+}+U_{-}\right)$and $L_{z}=2 T_{3}$. Using $L^{2}$ $=L_{z}^{2}-L_{z}+L_{+} L_{-}$, we find as before [27]

$$
\begin{aligned}
L^{2} & =4 T_{3}^{2}-2 T_{3}+2\left(V_{+} V_{-}+U_{-} U_{+}\right)+G_{Y} \\
& =4 T_{3}^{2}-2 T_{3}+2\left[n_{+}\left(1+n_{0}\right)+n_{0}\left(1+n_{-}\right)\right]+G_{Y},
\end{aligned}
$$

with $G_{Y}=2\left(V_{+} U_{+}+\right.$H.c. $)$. Using $n_{0}=N / 3-Y$ and $n_{ \pm}$ $=N / 3+Y / 2 \pm T_{3}$, we obtain

$$
L^{2}=4 T_{3}^{2}+\frac{1}{2}\left(N-\epsilon_{+}\right)\left(N-\epsilon_{-}\right)-2\left(Y-Y_{0}\right)^{2}+G_{Y}
$$

with $\epsilon_{ \pm}=-3 / 2 \pm \sqrt{2}$ and $Y_{0}=-N / 6-1 / 4$. For $N \gg 1$ we have $Y_{0}^{-} \approx-N / 6$ which corresponds to $n_{0}=N / 2$ independent of $n_{-}$and $n_{+}$. The point $Y_{0}$ acts as an attractor in the diffusive random-walk process described by the $G_{Y}$ term. Using $\left[T_{3}, V_{ \pm}\right]= \pm V_{ \pm} / 2$ and $\left[T_{3}, U_{ \pm}\right]=\mp U_{ \pm} / 2$ we check that $\left[T_{3}, G_{Y}\right]=0$ which is consistent with $\left[H, L_{z}\right]=0$. The effect of $G_{Y}$ is thus to simply change $Y$. If we consider simultaneous eigenstates of the commuting operators $N, Y, T_{3}$ as $\left|N, T_{3}, Y\right\rangle$, the action of $G_{Y}$ can be summarized as

$$
\begin{aligned}
V_{+}\left|N, T_{3}, Y\right\rangle= & \sqrt{\left(\frac{N}{3}-Y\right)\left(\frac{N}{3}+T_{3}+\frac{Y}{2}+1\right)} \\
& \times\left|N, T_{3}+\frac{1}{2}, Y+1\right\rangle,
\end{aligned}
$$




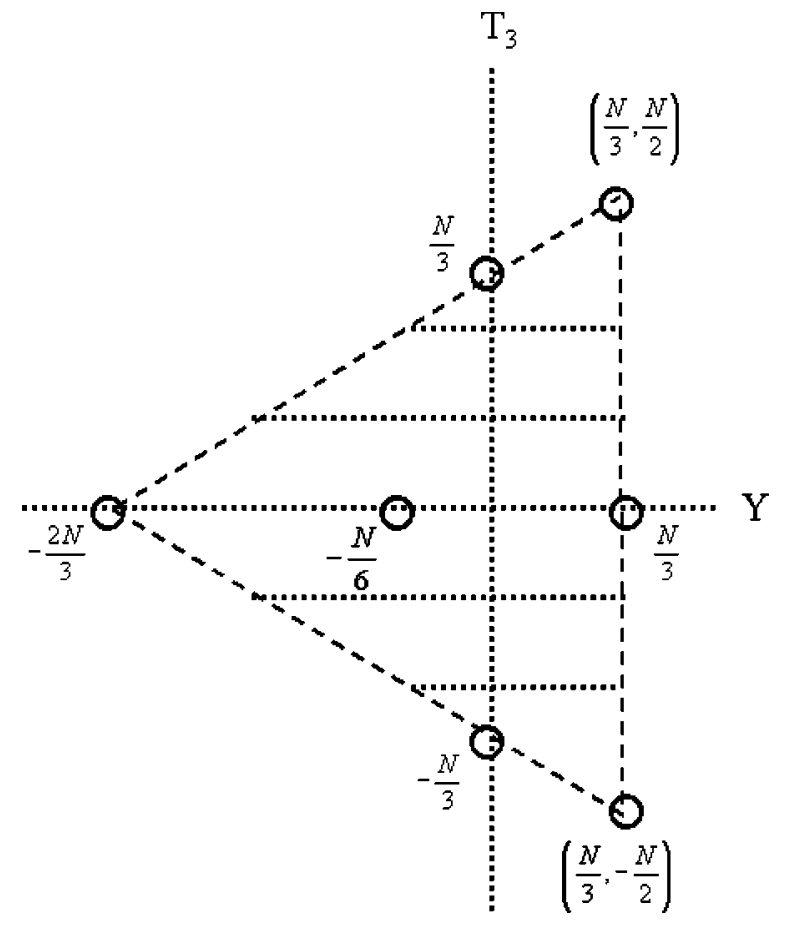

FIG. 1. The SU(3) $Y-T_{3}$ domain for a given $N$. The points within the triangular boundary constitute a set of applicable $\left(T_{3}, Y\right)$ pairs in the $\left(N, T_{3}, Y\right)$ triplets. By further fixing the $T_{3}$ block as it is also a constant of motion, the dynamics is reduced to the problem on a chain of $Y$ with reduced size as $T_{3}$ increases towards $\pm N / 2$.

$$
\begin{aligned}
U_{+}\left|N, T_{3}, Y\right\rangle= & \sqrt{\left(\frac{N}{3}-Y\right)\left(\frac{N}{3}-T_{3}+\frac{Y}{2}+1\right)} \\
& \times\left|N, T_{3}-\frac{1}{2}, Y+1\right\rangle .
\end{aligned}
$$

It couples the next-nearest neighbors along the $Y$ axis through an off-axial hopping in the $Y-T_{3}$ plane as illustrated in Fig. 1.

The three-mode Hamiltonian (3) can now be expressed as

$$
H=\sum_{\alpha} A_{\alpha} X_{\alpha}+\sum_{\alpha, \beta} A_{\alpha \beta} X_{\alpha} X_{\beta}+\left(g V_{+} U_{+}+\text {H.c. }\right),
$$

where $\alpha, \beta=N, T, Y ; X_{\alpha}=\left\{N, T_{3}, Y\right\}$, and $A_{\alpha \beta}$ are known functions of $g_{i j}^{s, a}$ as given in the Appendix. After dropping constant terms of $N$ and $T_{3}$, we obtain

$$
H=A_{y y}\left(Y-Y_{0}\right)^{2}+\left(g V_{+} U_{+}+\text {H.c. }\right),
$$

with

$$
Y_{0}=-\frac{A_{y}+A_{n y} N+A_{y t} T_{3}}{2 A_{y y}},
$$

and $c_{\alpha, \beta}$ as given in the Appendix. It turns out that the main difference from the previous decomposition of $L^{2}$ Eq. (13) (under the SMA) is the dependence of $Y_{0}$ on $T_{3}$. Using Eqs. (14) and (15), and denoting $\left|N, T_{3}, Y\right\rangle \equiv|Y\rangle$, we arrive at a Hamiltonian of the form

$$
H=\sum_{y}\left[A_{y y}\left(Y-Y_{0}\right)^{2}|Y\rangle\langle Y|+\left(g \Lambda_{y}|Y+2\rangle\langle Y|+\text { H.c. }\right)\right]
$$

with

$$
\Lambda_{y}=\sqrt{\left(\frac{N}{3}-Y\right)\left(\frac{N}{3}+\frac{Y}{2}+1\right)\left[\left(\frac{N}{3}+\frac{Y}{2}+1\right)-T_{3}^{2}\right]} .
$$

We note that the domain of the $\mathrm{SU}(3)$ basis $\left|N, T_{3}, Y\right\rangle$ is determined by

$$
\begin{gathered}
-\frac{2 N}{3}+2\left|T_{3}\right| \leqslant Y \leqslant \frac{N}{3}, \\
-\frac{N}{2} \leqslant T_{3} \leqslant \frac{N}{2},
\end{gathered}
$$

as illustrated in Fig. 1. For given $N$ and $T_{3}$, the line parallel to the $Y$ axis, crossing the triangle consists of the domain to determine the basis vectors of the system. As $T_{3}$ approaches its extremes, the number of basis vectors reduces to one. It can be seen that the length of the line along which the system dynamics occurs depends on $T_{3}$, and as such $T_{3}$ modifies the symmetry point and the fragmentation dynamics.

The eigenequation $E_{Y} \psi(Y)=H \psi(Y)$ for $\psi$ $=\Sigma_{Y} \phi(Y)|Y\rangle$ then becomes

$$
\begin{aligned}
E_{Y} \phi(Y)= & A_{y y}\left(Y-Y_{0}\right)^{2} \phi(Y)+g \Lambda_{y-2} \phi(Y-2) \\
& +g * \Lambda_{y} \phi(Y+2) .
\end{aligned}
$$

Assuming $|Y| \gg 2$ as the continuum limit in which $\phi(Y$ $\pm 2) \approx \phi(Y) \pm 2 \partial \phi(Y) / \partial Y+2 \partial^{2} \phi(Y) / \partial Y^{2}$, we obtain

$$
\begin{aligned}
E_{Y} \phi= & A_{y y}\left(Y-Y_{0}\right)^{2}+\left(g \Lambda_{y-2} \phi+g * \Lambda_{y}\right) \phi \\
& +2\left(g \Lambda_{y-2}-g^{*} \Lambda_{y}\right) \frac{\partial \phi}{\partial Y}+2\left(g \Lambda_{y-2}+g * \Lambda_{y}\right) \frac{\partial^{2} \phi}{\partial Y^{2}} .
\end{aligned}
$$

After tedious calculations, a simple analytical solution can be obtained if the coefficients of the differential terms become independent of $Y$. In the limit when $N / 3 \gg|Y|$ and $\left|T_{3}\right|$, we find

$$
E \phi(Y)=\frac{d^{2} \phi(Y)}{d Y^{2}}+\kappa \frac{d \phi(Y)}{d Y}+\varpi\left(Y-Y_{0}\right)^{2} \phi(Y),
$$

where

$$
\begin{gathered}
E=\frac{9 E_{Y}}{4 g N^{2}}-\frac{1}{2}, \\
\kappa=-\frac{3}{2 N},
\end{gathered}
$$




$$
\varpi=\frac{9 A_{y y}}{4 g N^{2}},
$$

are found under the assumption of $g \in \mathbb{R}$, (i.e., when $\delta_{+}$ $\left.+\delta_{-}-2 \delta_{0}=0, \pi\right)$. We see that $\kappa<0$ and the sign of $\varpi$ depends on the sign of $A_{y y}$. In contrast, $\varpi$ depends only on $N$ under the SMA [27].

The quantum dynamics for the three-mode model can now be investigated using the set of operator equations $i \partial X / \partial t=[X, H]$. Explicitly, we find

$$
\begin{gathered}
i \frac{d}{d t} Y=2\left(g V_{+} U_{+}-g * V_{-} U_{-}\right), \\
i \frac{d}{d t} V_{+}=c_{v} V_{+}+c_{v t} V_{+} T_{3}+c_{v y} V_{+} Y+c_{v n} V_{+} N \\
+g *\left(V_{-} T_{+}+2 V_{3} U_{-}\right), \\
i \frac{d}{d t} U_{+}=c_{u} U_{+}+c_{u t} U_{+} T_{3}+c_{u y} U_{+} Y+c_{u n} U_{+} N \\
+g^{*}\left(T_{-} U_{-}+2 V_{-} U_{3}\right) \\
i \frac{d}{d t} T_{+}=c_{t} T_{+}+c_{t t} T_{+} T_{3}+c_{t y} T_{+} Y+c_{t n} T_{+} N \\
+g V_{+}^{2}-g * U_{-}^{2}, \\
\frac{d}{d t} U_{3}=\frac{d}{d t} V_{3}=\frac{3}{4} \frac{d}{d t} Y .
\end{gathered}
$$

Both $N$ and $T_{3}$ are constants of motion as noted earlier, i.e., $d N / d t=d T_{3} / d t=0$. The coefficients $c_{x y}$ are linear combinations of the overlap integrals $g_{i j}^{a, s}$ as given in the Appendix. When the differences between overlap integrals are small one can further neglect several terms from the above equations. By employing the decorrelation (neoclassical) approximation [28] and taking into account the small parameters and the SU(3) group structure the resulting dynamics can be solved. In the simplest treatment one can also simply replace the operators by $c$ numbers, i.e., taking $\left\langle X_{i} X_{j}\right\rangle$ $=\left\langle X_{i}\right\rangle\left\langle X_{j}\right\rangle$. We note that Eq. (26) can be generalized to include external optical [29] and magnetic $[15,30]$ fields.

\section{SEMICLASSICAL DYNAMICS OF FRAGMENTATION}

Since we adopt a frozen spatial mode to explore the quantum dynamics, we will focus on the (position diagonal) single-particle local density-matrix operator $\rho_{i j}(\vec{r})$ $=\psi_{i}^{\dagger}(\vec{r}) \psi_{j}(\vec{r})$. Its matrix element is give by $\left\langle\rho_{i j}\right\rangle$ $=f_{i j}\left\langle a_{i}^{\dagger} a_{j}\right\rangle$ with the overlap integral

$$
f_{i j}=\int d \vec{r} \phi_{i}^{*}(\vec{r}) \phi_{j}(\vec{r}) \text {. }
$$

After solving for time evolution of the SU(3) operators, we obtain the effective single-particle local density matrix as given by

$$
\rho=\left(\begin{array}{ccc}
N / 3+Y / 2+T_{3} & f_{+0} V_{+} & f_{+-} T_{+} \\
f_{0+} V_{-} & N / 3-Y & f_{0-} U_{-} \\
f_{-+} T_{-} & f_{-0} U_{+} & N / 3+Y / 2-T_{3}
\end{array}\right)
$$

All off-diagonal terms in $\rho$ individually change $T_{3}$, yet $T_{3}$ remains a constant of motion. The off-diagonal terms have the property $\left\langle N^{\prime} Y^{\prime} T_{3}\left|X_{ \pm}\right| N Y T_{3}\right\rangle=0$ for $X=U, V, T$. This leads to the conclusion that $\langle\rho\rangle$ should be diagonal at all times for an initial state with a fixed $T_{3}$. The next-neighbor hopping induced by $G_{Y}$ changes the diagonal elements in time through their dependence on $Y$. The initial population distribution on the $Y$ axis also redistributes in time due to both the next-neighbor hopping and the "attractor" term $\left(Y-Y_{0}\right)^{2}$. Thus as long as the off-diagonal terms are absent, the fragmentation of an initially unfragmented condensate can be simply interpreted as a mode mixing or spin mixing process [12].

When the initial state is a superposition of states with different $T_{3}$, the off-diagonal elements of $\rho$ become significant, and the dynamics becomes more complicated. Situations could arise where atomic populations in all three Zeeman levels are macroscopic yet the system remains unfragmented [3]. In order to investigate fragmentation in such cases, it is necessary to determine the time evolution of all $U, V, T$ spin components and to find the eigenvalues $\Pi_{1,2,3}$ of $\rho$ at all times. From an experimental point of view this task is almost impossible as the $U, V$, and $T$ spin components are noncommuting and cannot be measured simultaneously. It is therefore impossible to construct atom-interferometric schemes to measure quadratures corresponding to both real and imaginary parts of $U_{+}, V_{+}, T_{+}$simultaneously within the same experiment. When the off-diagonal elements are large, there are basically five parameters, corresponding to the two phase differences between three spin components of $U, V, T$ and three amplitudes, enough to characterize $\rho$ completely. In this limit, it becomes possible to construct a set of five commuting observables [31]. To examine this situation, we follow Ref. [32] and consider the semiclassical dynamics by treating the $\mathrm{SU}(3)$ operators as $c$ numbers.

In the numerical study to be presented below, we first compute the set of overlap integrals by solving the three coupled GPEs with two undetermined Lagrange multipliers for the conservation of the total atom number and the magnetization [23]. Using these overlap integrals, we determine the interaction coefficients $E_{j}, g_{i j}^{a, s}$, and $g$ as well as parameters $A_{x y}$ and $c_{x y}$. We then numerically integrate the $c$-number version of Eq. (26), and construct and diagonalize the corresponding single-particle density matrix $\rho$ at different times and evaluate its eigenvalues. The overlap integrals

$$
I_{i j k l}=\int d \vec{r} \phi_{i}^{*} \phi_{j}^{*} \phi_{k} \phi_{l}
$$

for a typical simulation are summarized in Table I. We assume a ${ }^{23} \mathrm{Na}$ condensate (antiferromagnetic) with $N=10^{6}$ atoms, and scattering lengths $a_{2}=50\left(a_{B}\right)$ and $a_{0}=55\left(a_{B}\right)$ in units of Bohr radius $a_{B}$. We also assume a harmonic, 
TABLE I. The values of overlap integrals used in our numerical simulation of the fragmentation dynamics for a condensate with a zero magnetization $\left(T_{3}=0\right)$ and $N=10^{6}$. A condensate of ${ }^{23} \mathrm{Na}$ atom is confined in a harmonic and spherically symmetric trap with a radial trap frequency $\omega_{r}=(2 \pi) 100(\mathrm{~Hz})$ and an aspect ratio that corresponds to $\omega_{z} / \omega_{r}=1$.

\begin{tabular}{lccccccc}
\hline \hline$i j k l$ & ++++ & 0000 & ---- & ++-- & ++00 & --00 & +-00 \\
$I_{i j k l}$ & 0.0009 & 0.0009 & 0.0009 & 0.0009 & 0.0009 & 0.0009 & -0.0009 \\
\hline \hline$i j$ & $0.2909+0.9568 i$ & $0.8767+0.4809 i$ & $-0.7762+0.6304 i$ \\
$f_{i j}$ & \multirow{2}{*}{+0} & $0-$ & -+ \\
\hline \hline
\end{tabular}

spherically symmetric optical trap with a radial trap frequency $\omega_{r}=(2 \pi) 100(\mathrm{~Hz})$ and an aspect ratio corresponds to $\omega_{z} / \omega_{r}=1$.

The various values of the overlap integrals as presented in Table I reflect the fact that single-mode approximation is exact for ferromagnetic interactions, and remains true for the antiferromagnetic case provided that the condensate magnetization is zero [23]. The general model with three distinct modes developed here is applicable for any given mode functions and thus applies to the case of antiferromagnetic condensates with nonzero magnetization as well. Using these parameters, we propagate a system initially prepared in a coherent state of equally populated spin components $\left(n_{ \pm, 0}\right.$ $=N / 3)$, i.e., $|\psi\rangle=\left(a_{+}^{\dagger}+a_{0}^{\dagger}+a_{-}^{\dagger}\right)^{N}|\operatorname{vac}\rangle /\left(\sqrt{N !} \sqrt{3}^{N}\right)$ with all parameters being real. Such a state can be realized using Raman coupling with external laser pulses [12]. With time, the initial fragmented state becomes unfragmented as two eigenvalues of the single-particle density matrix diminish while the third one gets close to $N$. This exchange of fragmented and unfragmented states occurs periodically in time within the semiclassical dynamics as shown in Fig. 2 for $N$ $=10^{6}$ atoms. We expect that quantum character of the model system will change this picture, especially in the limit when the number of atoms is small where use of the semiclassical approach becomes questionable. In the following section, we

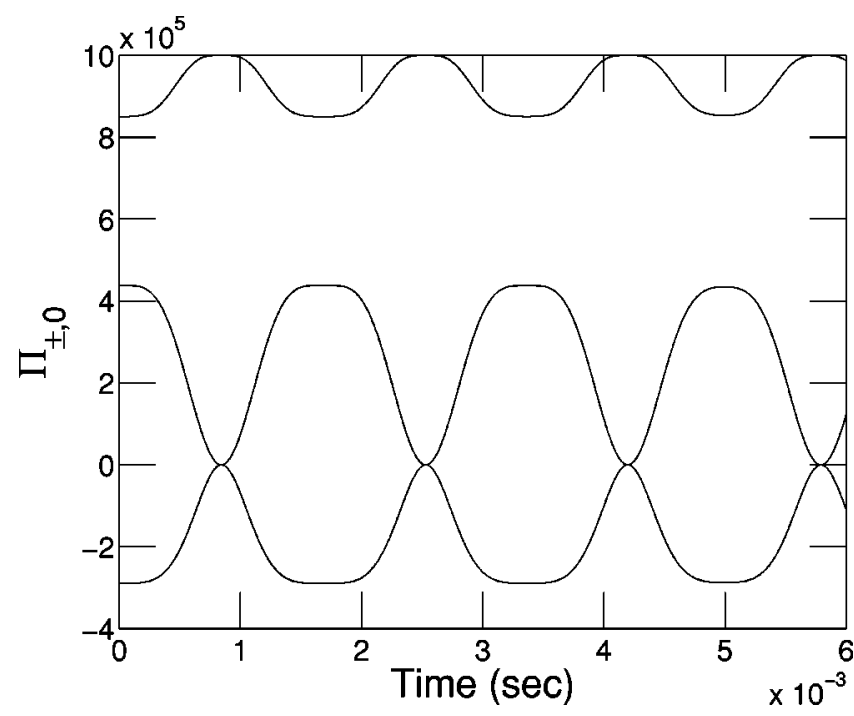

FIG. 2. The time-dependent eigenvalues of the single particle density matrix $\rho$ for $N=10^{6}\left({ }^{23} \mathrm{Na}\right)$ atoms. The condensate is in a coherent state with $n_{ \pm, 0}=N / 3$ initially . shall exploit the fact that the SMA works well at small numbers of atoms and determine the eigenvalues in a full quantum-mechanical treatment.

\section{QUANTUM DYNAMICS OF FRAGMENTATION}

We now explore the quantum dynamics and look for signatures of fragmentation in the population oscillations as well as in the two-particle correlations in terms of population variances. To this end, we start with an initial state that is prepared by optical Raman processes in a state described by [12]

$$
|\psi(0)\rangle=\frac{1}{\sqrt{N !}}\left(\alpha_{0} a_{0}^{\dagger}+\alpha_{-} a_{-}^{\dagger}+\alpha_{+} a_{+}^{\dagger}\right)^{N}|0,0,0\rangle,
$$

where $|0,0,0\rangle$ is the vacuum state in the Fock basis of $\left|n_{0}, n_{-}, n_{+}\right\rangle$and $\alpha_{j}=\left|\alpha_{j}\right| \exp \left(i \delta_{j}\right)$ are complex numbers. Using $N=n_{0}+n_{+}+n_{-}, m=n_{+}-n_{-}$, and the combinatorial symbol

$$
C_{N}^{m}=\frac{N !}{m !(N-m) !},
$$

we can express the initial state as

$$
|\psi(0)\rangle=\sum_{k} \psi_{N m k}(\vec{\alpha})\left|2 k, \frac{N-m}{2}-k, \frac{N+m}{2}-k\right\rangle,
$$

where

$$
\psi_{N m k}=\sqrt{C_{N}^{2 k} C_{N-2 k}^{(N-m) / 2-k}} \alpha_{0}^{2 k} \alpha_{-}^{(N-m) / 2-k} \alpha_{+}^{(N+m) / 2-k},
$$

with $\vec{\alpha}=\left(\alpha_{0}, \alpha_{-}, \alpha_{+}\right), k=0,1, \ldots,(N-|m|) / 2$ for even $N$ $+m$ and $k=1 / 2,3 / 2, \ldots,(N-|m|) / 2$ for odd $N+m$. The transformation coefficients between angular momentum basis and the Fock basis were found in Ref. [33], which can be expressed in a more compact form as

$$
\begin{gathered}
|l m\rangle=\sum_{k} G_{l m k}\left|2 k, \frac{N-m}{2}-k, \frac{N+m}{2}-k\right\rangle, \\
G_{l m k}=2^{k} s_{l} \sum_{r} \frac{(-1)^{r}}{4^{r}}\left(\begin{array}{c}
N l m \\
k r
\end{array}\right),
\end{gathered}
$$

where we introduced a binomial symbol such that 

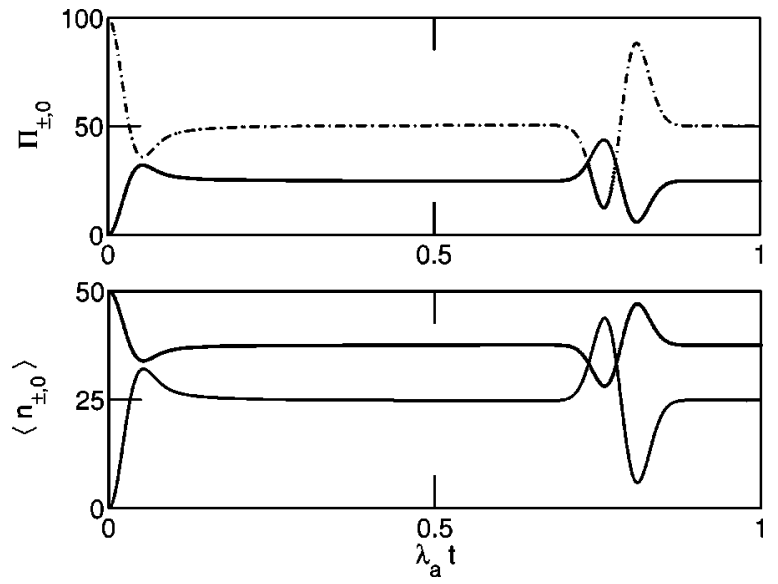

FIG. 3. The evolution of eigenvalues for $\rho$ from an initial state with $\delta=0, P_{0}=0$, and $N=100$. The lower curve in the bottom figure denotes $\left\langle n_{0}\right\rangle$.

$$
\begin{aligned}
& \left(\begin{array}{c}
N l m \\
k r
\end{array}\right) \\
& \quad=\sqrt{\frac{C_{2 r}^{r} C_{2 k}^{2 r} C_{l}^{2 k-2 r} C_{N-2 k}^{l-2 k+2 r} C_{N-l-2 r}^{(N-l) / 2-r}}{C_{N}^{(N-m) / 2-k} C_{2 l}^{l-m}} C_{l-2 k+2 r}^{(l-m) / 2-k+r} .}
\end{aligned}
$$

We note $l=N, N-2, \ldots, N-2[N / 2]$ with $[n]=n$ or $n$ $-1 / 2$ for $n=$ even or odd, and $r=\max [0, k-(l$ $-|m|) / 2], \ldots, \min [k,(N-l) / 2], \quad m=0, \pm 1, \pm 2, \ldots, \pm l . \quad k$ $=0,1,2, \ldots,(N-|m|) / 2$ for $l+m=$ even and $k$ $=1 / 2,3 / 2, \ldots,(N-|m|) / 2$ for $l+m=$ odd. The normalization is given by

$$
s_{l}=\left[\sum_{j=0}^{(N-l) / 2} \frac{1}{4^{j}} C_{2 j}^{j} C_{(N+l) / 2-j}^{j}\right]^{-1 / 2} .
$$

We will use an initial state of the same form as used in Ref. [12] with $\alpha_{0}=\sqrt{P_{0}} \exp (i \delta / 2)$ and $\alpha_{ \pm}=\sqrt{1-P_{0}}$.
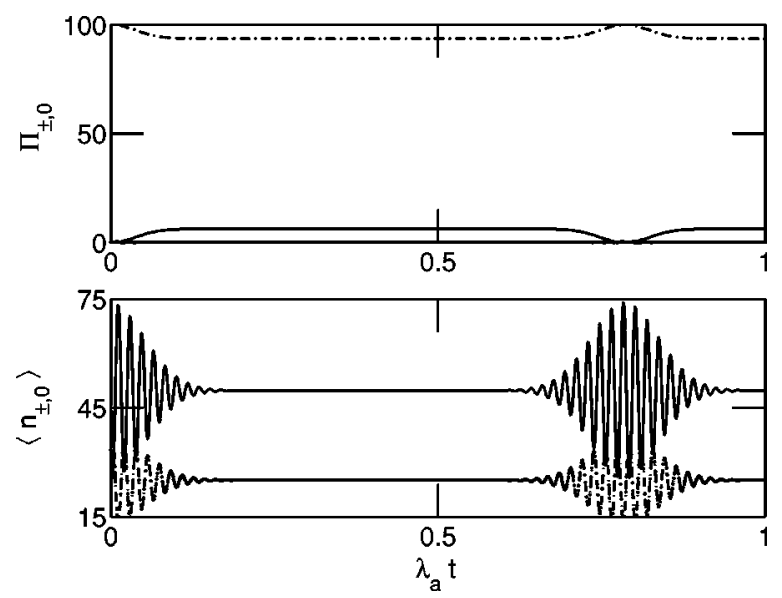

FIG. 4. The upper figure shows the evolution of eigenvalues for $\rho$ from an initial state with $\delta=\pi / 8, P_{0}=1 / 2$, and $N=100$. The upper curve in the bottom figure is for $\left\langle n_{0}\right\rangle$.

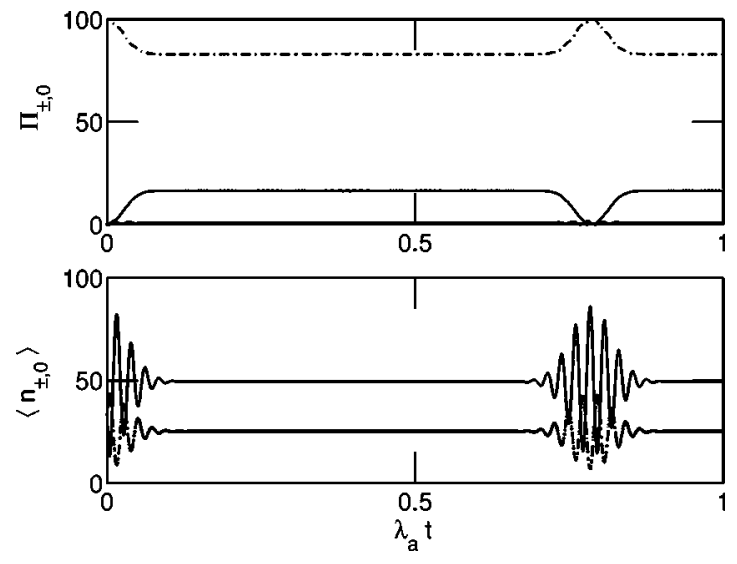

FIG. 5. The same as Fig. 4, but for $\delta=\pi / 4$.

It is now straightforward to propagate this initial state using the three-mode Hamiltonian (16) and to determine the eigenvalues of the single-particle density-matrix operator $\rho$ at all times. We have performed this for an initial state with $\Pi_{1,2}=0$ and $\Pi_{3}=N$, an unfragmented state for any choice of Raman pulse configurations. With the time evolution, however, the situation changes drastically. For the simpler case of only two populated modes with $P_{0}=0$, Fig. 3 demonstrates that there exists an extended temporal region where the condensate becomes fragmented. These regions are in between the collapse and revival regions of spin component population oscillation. At the end of collapse region, fragmentation occurs with $\Pi_{j} \sim N / 3$. The two mode results are independent of the phase difference $\delta$. However, when the $m_{f}=0$ mode is also populated, we find $\delta$ plays an important role. In Figs. 4-7, $P_{0}$ is kept constant at $1 / 2$ while the phase difference varied for $\delta=\pi / 8, \pi / 4$, and $\pi / 2$, respectively. The fragmentation is found to increase with $\delta$ when $P_{0}$ is kept constant. When $P_{0}=1 / 2$ and $\delta=0$, the case for a stationary state, we find however, $\Pi_{1}=N$ and $\Pi_{2,3}=0$. By changing $\delta$, this stationary initial state is indeed observed to dynamically develop into a fragmented condensate.

It was argued in Ref. [3] that while the single-particle density matrix probes the existence of fragmentation, the characterization of fragmentation requires consideration of

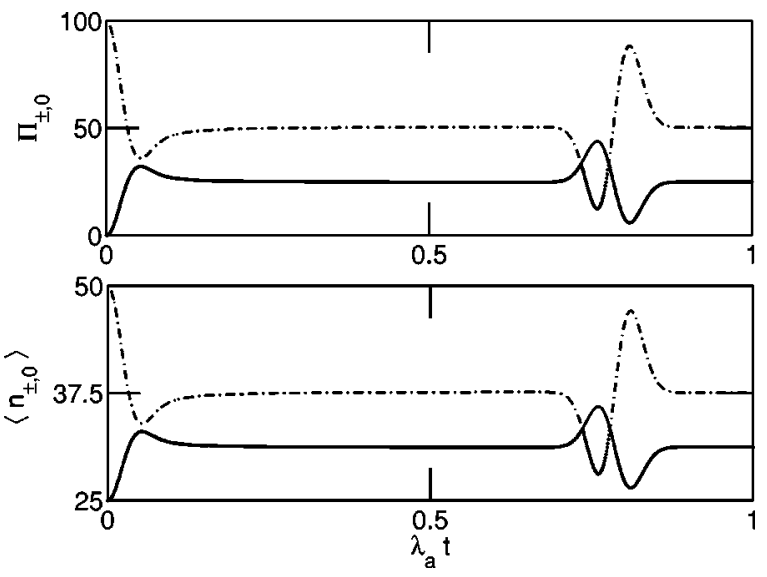

FIG. 6. The same as Fig. 4, but for $\delta=\pi / 2$. 

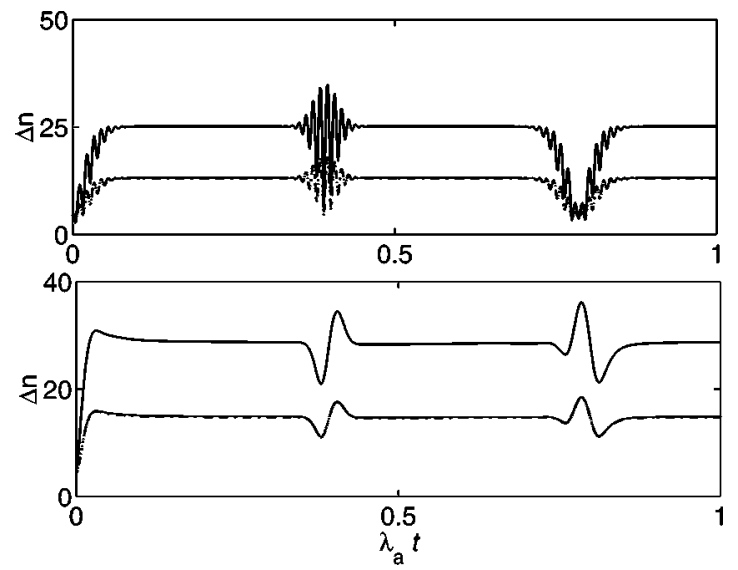

FIG. 7. Atom-number fluctuations for each internal Zeeman state when $P_{0}=1 / 2$, and $\delta=\pi / 4$ (upper figure) or $\delta=\pi / 2$ (lower figure). The upper curves in both figures are for fluctuations in $n_{0}$.

higher-order particle correlations. In particular, two particle correlations can be used to distinguish the two extreme regimes of fragmentation: the superfragmented regime and the coherent fragmented regime. When the atom-number fluctuation $\left(\Delta n_{i}\right)^{2}=\left\langle\left(a_{i}^{\dagger} a_{i}\right)^{2}\right\rangle-\left\langle a_{i}^{\dagger} a_{i}\right\rangle^{2}$ is of the same order as number of particles $(N)$, the system is said to be superfragmented; when $\Delta n_{i} \sim 1$, it is called coherent fragmented. We have calculated $\Delta n_{i}$ for $P_{0}=1 / 2$ and $\delta=\pi / 4, \pi / 2$. The results are shown in Fig. 7. We find that while the system remains in between these two extreme regimes at all times, it is closer to the coherent fragmented regime.

\section{CONCLUSION}

We have shown that fragmentation of a spin- 1 condensate can be optically induced and controlled by both the amplitudes and phase differences of Raman pulses. We have investigated the quantum dynamics (due to coherent atomic collisions) of such optical Raman engineered initial states with only one macroscopic eigenvalue of the single-particle density matrix, i.e., initially unfragmented condensates. During the course of the dynamic evolution, we find that the initial macroscopic eigenvalue decreases while the other two increase. For certain initial configurations, arranged by the phases and amplitudes of the Raman lasers, all three eigenvalues can be made comparable. In other cases, all three eigenvalues remain close to their initial values. We have studied quantum dynamics, taking into account the different spatial modes for the different condensate spin component, i.e., we have performed these quantum dynamics investigations in the regime beyond the usual SMA [12]. While it is difficult to perform a tomographic or atom-interferometric reconstruction of the single-particle density matrix, it is in principle possible for our system because of the existence of commuting Stokes parameters for a three-mode bosonic system [31]. For our purpose in this work, however, we have found an easier approach associated with the phenomenon of quantum fragmentation. We find that, for the initial states with a zero magnetization as considered here, atomic population oscillations between the collapse and revival regions disappear whenever fragmentation occurs. We also computed population fluctuations (two-particle correlations), a characterization of the type of fragmentation regime. Our numerical results indicate that the system as studied here is closer to the coherent fragmented regime at all times. As in the case of Ref. [12], one of the major experimental challenges to confirm our results is to devise a careful and precise interferometric arrangement of Raman pulses for imprinting the relative phase $\delta$ on the initially unfragmented spin- 1 condensate.

\section{ACKNOWLEDGMENTS}

We acknowledge helpful discussions with Dr. M. Barrett. C. P. Sun acknowledges the support of CNSF, the Knowledge Innovation Program (KIP) of the Chinese Academy of Science, and the National Fundamental Research Program of China (Grant No. 001GB309310). M. Zhang, S. Yi, and L. You acknowledge the support of NSF.

\section{APPENDIX: INTERACTION COEFFICIENTS}

We list the various coefficients used in the paper in this appendix. The first set is for the three-mode Hamiltonian as in Eq. (16), they are given by

$$
\begin{aligned}
& A_{n}=\frac{1}{6}[\left.2\left(E_{+}+E_{0}+E_{-}\right)-g_{++}^{s}-g_{00}^{s}-g_{--}^{s}-g_{++}^{a}-g_{--}^{a}\right], \\
& A_{t}= \frac{1}{2}\left[2\left(E_{+}-E_{-}\right)-g_{++}^{s}+g_{--}^{s}-g_{++}^{a}+g_{--}^{a}\right], \\
& A_{y}=\frac{1}{4}\left[2\left(E_{+}+E_{-}-2 E_{0}\right)-g_{++}^{s}-g_{--}^{s}-g_{++}^{a}-g_{--}^{a}\right. \\
&\left.+2 g_{00}^{s}\right], \\
& A_{n n}=\frac{1}{18}\left[g_{++}^{s}+g_{++}^{a}+g_{--}^{s}+g_{--}^{a}+g_{00}^{s}\right. \\
&\left.\quad+2\left(g_{+-}^{s}-g_{+-}^{a}+g_{+0}^{s}+g_{+0}^{a}+g_{-0}^{s}+g_{-0}^{a}\right)\right],
\end{aligned}
$$

$$
\begin{gathered}
A_{n t}=\frac{1}{3}\left[g_{++}^{s}+g_{++}^{a}-g_{--}^{s}-g_{--}^{a}+g_{+0}^{s}+g_{+0}^{a}-g_{-0}^{s}-g_{-0}^{a}\right], \\
A_{n y}=\frac{1}{6}\left[g_{++}^{s}+g_{++}^{a}+g_{--}^{s}+g_{--}^{a}\right. \\
\left.+2\left(g_{+-}^{s}-g_{+-}^{a}-g_{+0}^{s}-g_{+0}^{a}-g_{-0}^{s}-g_{-0}^{a}-g_{00}^{s}\right)\right], \\
A_{t t}=\frac{1}{2}\left[g_{++}^{s}+g_{++}^{a}+g_{--}^{s}+g_{--}^{a}+2\left(g_{+-}^{a}-g_{+-}^{s}\right)\right], \\
A_{t y}=\frac{1}{2}\left[g_{++}^{s}+g_{++}^{a}-g_{--}^{s}-g_{--}^{a}\right. \\
\left.\quad+2\left(g_{-0}^{s}+g_{-0}^{a}-g_{+0}^{s}-g_{+0}^{a}\right)\right], \\
A_{y y}=\frac{1}{8}\left[g_{++}^{s}+g_{++}^{a}+g_{--}^{s}+g_{--}^{a}+2\left(2 g_{00}^{s}+g_{+-}^{s}-g_{+-}^{a}\right.\right. \\
\left.\left.-2 g_{+0}^{s}-2 g_{+0}^{a}-2 g_{-0}^{s}-2 g_{-0}^{a}\right)\right] .
\end{gathered}
$$

The next set of coefficients are for the operator Eq. (26):

$$
\begin{gathered}
c_{u}=\frac{A_{t}}{2}-A_{y}-A_{y y}-\frac{A_{t t}}{4}+\frac{A_{y t}}{2}, \\
c_{u t}=A_{t t}-A_{t y},
\end{gathered}
$$




$$
\begin{gathered}
c_{u y}=-2 A_{y y}+\frac{A_{t y}}{2}, \\
c_{u n}=\frac{A_{n t}^{s}+A_{n t}^{a}}{2}-\left(A_{n y}^{s}+A_{n y}^{a}\right), \\
c_{v}=-\frac{A_{t}}{2}-A_{y}-A_{y y}-\frac{A_{t t}}{4}-\frac{A_{y t}}{2}, \\
c_{v t}=-A_{t y}-A_{t t}, \\
c_{v y}=-2 A_{y y}-\frac{A_{t y}}{2},
\end{gathered}
$$

$$
\begin{gathered}
c_{v n}=-\frac{A_{n t}^{s}+A_{n t}^{a}}{2}-\left(A_{n y}^{s}+A_{n y}^{a}\right), \\
c_{t}=-A_{t}-A_{t t}, \\
c_{t t}=-2 A_{t t}, \\
c_{t y}=-A_{t y}, \\
c_{t n}=-A_{n t} .
\end{gathered}
$$

[1] C.J. Pethick and L.P. Pitaevskii, Phys. Rev. A 62, 033609 (2000); N.K. Wilkin, J.M.F. Gunn, and R.A. Smith, Phys. Rev. Lett. 80, 2265 (1997); B. Mottelson, ibid. 83, 2695 (1998); O. Penrose and L. Onsager, Phys. Rev. 104, 576 (1956).

[2] P. Nozières and D.S. James, J. Phys. (Paris) 43, 1133 (1982); P. Nozières, in Bose-Einstein Condensation, edited by A. Griffin, D. Snoke, and S. Stringari (Cambridge University Press, Cambridge, 1995).

[3] T.-L. Ho and S.K. Yip, Phys. Rev. Lett. 84, 4031 (2000).

[4] D.M. Stemper-Kurn et al., Phys. Rev. Lett. 80, 2027 (1998).

[5] M. Barrett, J. Sauer, and M.S. Chapman, Phys. Rev. Lett. 87, 010404 (2001).

[6] T.-L. Ho, Phys. Rev. Lett. 81, 742 (1998).

[7] T. Ohmi and K. Machida, J. Phys. Soc. Jpn. 67, 1822 (1998).

[8] W. Zhang and D.F. Walls, Phys. Rev. A 57, 1248 (1998).

[9] C.K. Law et al., Phys. Rev. Lett. 81, 5257 (1998).

[10] E. Goldstein and P. Meystre, Phys. Rev. A 59, 3896 (1998).

[11] M. Koashi and M. Ueda, Phys. Rev. Lett. 84, 1066 (2000).

[12] H. Pu, C.K. Law, and N.P. Bigelow, Physica B 280, 27 (2000); H. Pu, S. Raghavan, and N.P. Bigelow, Phys. Rev. A 61, 023602 (2000); H. Pu, C.K. Law, S. Raghavan, J.H. Eberly, and N.P. Bigelow, ibid. 60, 1463 (1999).

[13] L.-M. Duan, J.I. Cirac, and P. Zoller, Phys. Rev. A 65, 033619 (2002).

[14] J. Dukelsky and P. Schuck, Phys. Rev. Lett. 86, 4207 (2001).

[15] H. Pu, S. Raghavan, and N.P. Bigelow, Phys. Rev. A 61, 023602 (2000).

[16] A.S. Sorensen, Phys. Rev. A 65, 043610 (2002).
[17] N.P. Robins, W. Zhang, E.A. Ostrovskaya, and Y.S. Kivshar, Phys. Rev. A 64, 021601(R) (2001).

[18] M. Ueda, Phys. Rev. A 63, 013601 (2000).

[19] M. Lewenstein, L. You, J. Cooper, and K. Burnett, Phys. Rev. A 50, 2207 (1994).

[20] G.J. Milburn, J. Corney, E.M. Wright, and D.F. Walls, Phys. Rev. A 55, 4318 (1997).

[21] M.J. Steel and M.J. Collett, Phys. Rev. A 57, 2920 (1998).

[22] J.E. Williams, Phys. Rev. A 64, 013610 (2001).

[23] S. Yi, Ö.E. Müstecaplıoğlu, C.P. Sun, and L. You, Phys. Rev. A 66, 011601(R) (2002).

[24] H. Pu, C.K. Law, S. Raghavan, J.H. Eberly, and N.P. Bigelow, Phys. Rev. A 60, 1463 (1999).

[25] M. Kitagawa and M. Ueda, Phys. Rev. A 47, 5138 (1993); D.J. Wineland et al., ibid. 50, 67 (1994).

[26] M. Gell-Mann, Phys. Rev. 92, 833 (1953); T. Nakuno and K. Nishijima, Prog. Theor. Phys. 10, 581 (1953).

[27] Ö.E. Müstecaplığlu, M. Zhang, and L. You, Phys. Rev. A 66, 033611 (2002).

[28] R. Bonifacio and A.L. Lugiato, Phys. Rev. A 12, 587 (1975).

[29] S. Raghavan, H. Pu, P. Meystre, and N.P. Bigelow, Opt. Commun. 188, 149 (2001).

[30] H. Pu, S. Raghavan, and N.P. Bigelow, Phys. Rev. A 63, 033603 (2001).

[31] A.S. Shumovsky and Ö.E. Müstecaplıoglu, Phys. Rev. Lett. 80, 1202 (1998).

[32] K. Nemoto, C.A. Holmes, G.J. Milburn, and W.J. Munro, Phys. Rev. A 63, 013604 (2000).

[33] Y. Wu, Phys. Rev. A 54, 4534 (1996). 